\title{
Electric dipole moments in supersymmetric theories
}

\author{
Andrea Romanino* \\ Dipartimento di Fisica, Università di Pisa and INFN, Sezione di \\ Pisa, I-56126 Pisa, Italy
}

\begin{abstract}
Intrinsic EDMs in microscopic systems at a level of sensitivity achievable in experiments under way or foreseen are predicted in supersymmetric unified theories. I describe this and other sources of measurable EDMs and I show how these sources can be distinguished through experiments in different systems.
\end{abstract}

The intrinsic dipole moments (EDMs) of microscopic systems constitute an observable of great interest because they can provide informations on CP violation and physics beyond the Standard Model. An improvement of the sensitivity of the present experiments by one or two orders of magnitude, as pursued by foreseen experiments, might give rise to a positive signal (Ramsey, 1995; DeMille, 1995). The present limits for the neutron (Altarev et al., 1992) and electron ${ }^{1}$ (Commins et al., 1994) EDM are shown below

$$
d_{n}<0.8 \cdot 10^{-25} e \mathrm{~cm} \quad d_{e}<4.3 \cdot 10^{-27} e \mathrm{~cm} .
$$

EDMs in this range are predicted as unavoidable effects ${ }^{2}$ in presence of supersymmetric unification with $\mathrm{SO}(10)$ as gauge group if the supersymmetry breaking is communicated to the observable fields at the Planck scale. From this point of view, supersymmetric unification differs from non unified and/or non supersymmetric models in which the CP violation is due to the CKM phase, that all predict much too small EDMs. A possible EDM signal in experiments in progress or foreseen could then represent, as $\mu \rightarrow e \gamma$ and other flavour violating processes (Barbieri and Hall, 1994; Barbieri et al., 1995b), an indirect signal of supersymmetric unification (Dimopoulos and Hall, 1995; Barbieri et al., 1995a). However, the same effect can also be generated in non unified theories, in particular in the Minimal Supersymmetric Standard Model (MSSM) if the soft supersymmetry breaking parameters are complex already at the Planck scale, or, more generally, if strong $\mathrm{CP}$ violation is present.

In this presentation, I mean first to discuss the origin of the large effect in unified supersymmetric models and then to consider the possibility of distinguishing experimentally among the quoted sources of CP violation at the level of present or foreseen experiments (in the following, I will use "observable effects" with this meaning).

\footnotetext{
*Email: romanino@ibmth.difi.unipi.it
}

${ }^{1}$ The EDM is measured in neutral systems; on the other side, the EDM of paramagnetic atoms is mainly due to the EDM of the unpaired electron (Barr, 1993; Fischler et al., 1992, and references therein) and this allows for an indirect determination of the electron EDM.

${ }^{2}$ Barring accidental cancellations. 


\section{EDMs in superunified models}

EDMs, as every signal of $\mathrm{CP}$ violation, require the presence of uneliminable phases in the lagrangian of the theory. In the Standard Model, the only phase is contained in the CKM matrix that appears in the charged current interactions expressed in terms of mass eigenstates. The effects of this CKM phase are largely unobservable: the prediction for the neutron EDM is $d_{n} \lesssim \mathcal{O}\left(10^{-33}\right) e \mathrm{~cm}$ (Shabalin, 1983).

In a generic supersymmetric extension of the Standard Model, the presence of new fields and couplings can involve the existence of new uneliminable phases. In particular they can arise when the couplings of quarks and leptons with their supersymmetric partners are expressed in terms of mass eigenstates and the corresponding transition matrices appear in the couplings.

Let us suppose that the soft supersymmetry breaking parameters are flavour universal and real at the Planck scale $M_{\mathrm{P}}$, as it happens in a large class of models in which the supersymmetry breaking is communicated to the observable sector by gravity (Barbieri et al., 1982; Chamseddine et al., 1982). In this case, at the Planck scale and at the tree level the scalar masses are degenerate in flavour, so no transition matrices appear in the fermion-sfermion couplings. However, the non universal couplings of the theory, namely the Yukawa matrices, correct radiatively the soft parameters, that lose in this way their universality. In particular, at the Fermi scale, where the EDMs are generated, the corrections due to the top Yukawa coupling, that we know to be large, are important. If the ratio of the vacuum expectation values of down and up Higgs is small (large $\tan \beta$ ), also the bottom Yukawa coupling can be important.

Is this enough in order to have observable EDMs? The answer depends on the theory structure below the Planck scale. More precisely, if the gauge group is the Standard Model one until the Planck scale, the effects are well below the experimentally accessible region, whereas, if an intermediate stage of unification is present between the Fermi scale and the Planck scale, observable effects can be generated both for the neutron and for the electron.

The reason for this difference is that in the former case the EDMs due to the radiatively induced fermion-sfermion mixing are vanishing when some of the light Yukawa couplings are vanishing, whereas this does not happen in the latter case. In fact, let us consider for example the EDM of the down quark in the limit of vanishing up and charm Yukawa couplings $\lambda_{u}=\lambda_{c}=0$. If the Standard Model gauge group holds until the Planck scale, in this limit there is no CP violation in the theory and the $d$ quark EDM is vanishing. In fact, at the Planck scale the soft parameters are universal and real by hypothesis and, after eliminating non physical phases from the CKM matrix, the physical one can be reached and eliminated with a $\mathrm{U}(2)$ rotation in the up-charm sector and a redefinition of field phases. On the other hand, if at the Planck scale the theory is unified, it is impossible to do a rotation or a phase redefinition only in the left sector, because this sector is unified with the up right one.

Let us look now more closely how EDMs for the $d$ quark and the electron arise in the case of $\mathrm{SO}(10)$ unification and moderate $\tan \beta$, in which the only important Yukawa is the top one $\lambda_{t}$. At the Planck scale and at the tree level, in the universality hypothesis the supersymmetry breaking mass term for the scalars of the three generations is $m_{\mathrm{P}}^{2} \tilde{1}_{i}^{\dagger} \tilde{1}_{i}$, where $\tilde{1}_{i}$ is the field that unifies the scalar-quarks and scalar-leptons of the $i$-th generation. As a consequence of radiative corrections due to $\lambda_{t}$, at the unification scale $M_{\mathrm{G}}$ the mass of all third generation scalars is different from the mass of other generations and, in the basis in which the Yukawa matrix that involve $\lambda_{t}$ is diagonal, the mass term becomes

$$
\sum_{i=1}^{2} m_{\mathrm{G}}^{2} \tilde{6}_{i}^{\dagger} \tilde{1}_{i}+\left(m_{\mathrm{G}}^{2}-\delta m_{\mathrm{G}}^{2}\right) \tilde{1}_{3}^{\dagger} \tilde{1}_{3}
$$

From the GUT scale to the Fermi scale, the scalar partners of the top, left and right, and of the bottom left are further corrected.

In the basis considered both the up and scalar up mass matrices are diagonal, whereas the down and charged lepton mass matrices are non diagonal. Therefore, the relative rotation between 
the mass eigenstates of fermions and scalars is $\mathbf{1}$ in the up sector (both left and right), whereas in the down and charged lepton sectors it is given by the CKM matrix ${ }^{3}$. The misalignment in the up sector and the resulting up quark EDM can be important in the large $\tan \beta$ case. In the present case only down quark and electron EDMs are generated in a significant way. In a wide region of parameters space, EDMs in the observable range are predicted for the neutron and the electron (Dimopoulos and Hall, 1995; Barbieri et al., 1995b; Barbieri et al., 1995a).

The leading contributions to the EDMs of $d$ and $e$ turn out to be proportional to the bottom and tau mass respectively through the insertion of the left-right block of the scalar mass matrices. They are also proportional to the imaginary part of a product of CKM matrix elements that involves $V_{t d}^{2}$. Moreover, they grow as $\lambda_{t}^{4}$ because the effect is a consequence of the splitting in the scalar mass matrices both in the left and right sector. More precisely, $d_{d}$ is subject to a GIM suppression if $\delta m_{\tilde{d}_{L}}^{2} / M_{3}^{2}$ or $\delta m_{\tilde{d}_{R}}^{2} / M_{3}^{2}$ is small, where $M_{3}$ is the gluino mass and $\delta m_{\tilde{d}_{L}}^{2}, \delta m_{\tilde{d}_{R}}^{2}$ are the 1-3 splittings in the mass of left and right down scalars, both proportional to $\lambda_{t}^{2}$. The contribution to $d_{e}$ is subject to the same kind of suppression if $\delta m_{\tilde{e}_{L}}^{2} / M_{2}^{2}$ or $\delta m_{\tilde{e}_{R}}^{2} / M_{2}^{2}$ is small, where this time $M_{2}$ is the $\mathrm{SU}(2)_{L}$ gaugino mass. Since at the Fermi scale $M_{3}^{2}$ is significantly greater than $M_{2}^{2}$, whereas the 1-3 splittings in the squark and slepton sectors are not so different, the GIM cancellation is more effective in suppressing the down EDM relative to the electron one.

\section{Comparison among different sources}

We saw that supersymmetric unification is a source of EDMs observable in experiments under way or foreseen. However, a signal, e.g., of a neutron EDM does not constitute "per se" an evidence of supersymmetric unification. As a matter of fact, also in the MSSM with universal soft terms, observable effects can arise if the soft supersymmetry breaking parameters are complex already at the Planck scale (Polkinski and Wise, 1983; del Aguila et al., 1983; Buchmuller and Wyler, 1983). In this case, we can have two further physical phases that, in a supersymmetric basis, are those of the B term and of the universal A term at the Planck scale (defined as the parameters that multiply the corresponding superpotential parameters in the bilinear and trilinear scalar interactions respectively). These phases give rise to large EDMs for the electron, the down quark and also the up quark, because they appear both in the left-right block of scalar mass matrices and in the gaugino-higgsino mass matrices. In this case the EDMs of $e, d$ and $u$ are proportional to their masses unlike the previous case where the fermion-sfermion mixing allowed to have third generations in the scalar loops. Moreover, another possibility that has to be considered is that the possible signal of an EDM is a strong CP violation effect.

In the event that EDMs will be measured in the near future, is therefore important to be able to distinguish among the described sources, the superunification, the presence of soft universal phases and strong $\mathrm{CP}$ violation. It turns out that the range of values that the neutron and electron EDMs $d_{n}, d_{e}$ can individually assume in the three cases are largely overlapping themselves, due to the unknown phases. On the other hand, the predictions for the ratio $d_{n} / d_{e}$ are characteristic of the different source (Barbieri et al., 1996).

Quite clearly, if the effect were due to strong $\mathrm{CP}$ violation, only the neutron would have a significant EDM. Moreover, in the case of EDMs generated by soft phases the $d_{n} / d_{e}$ ratio is greater than in the unification case for two reasons: first, as we saw, in the latter case there is a GIM cancellation that is more effective in suppressing $d_{d}$ and hence $d_{n}{ }^{4}$ relative to $d_{e}$. Furthermore, in the former case the fermion EDMs are proportional to their masses, whereas in the latter case

\footnotetext{
${ }^{3}$ More precisely, except for the down left sector, the rotation is given by the CKM matrix evaluated at the unification scale.

${ }^{4}$ In addition, in this case the up quark does not contribute to $d_{n}$, but, from a numerical point of view, this is not very important.
} 
they are proportional to the corresponding third generation fermions, so that

$$
\left(\frac{d_{n}}{d_{e}}\right)_{\substack{\text { soft } \\ \text { phases }}} /\left(\frac{d_{n}}{d_{e}}\right)_{\mathrm{GUT}} \propto \frac{m_{d}}{m_{e}} / \frac{m_{b}}{m_{\tau}} \approx 5 \pm 2 .
$$

These qualitative considerations have been made quantitative in a numerical analysis in which the contributions of chromoelectric dipole moments of quarks to the neutron EDM are taken in account (the Weinberg three-gluon operator and four-fermion operators do not contribute in a significant way) and the parameters are made to vary in a random way (Barbieri et al., 1996). From this analysis it is confirmed that $d_{n}$ or $d_{e}$ generated by soft phases are generally not consistent with the present experimental limits in most of relevant parameter space unless the phases are small, whereas this is not the case in superunification. Irrespective of the values of the phases, the $d_{n} / d_{e}$ ratio is actually smaller in the unification case: $0.12 \cdot 10^{ \pm 0.83}$ against $4.2 \cdot 10^{ \pm 0.34}$ in the other case $\mathrm{e}^{5}$. The two predictions are closer when the gluino is light, because in this region the GIM suppression of $d_{d}$ fails.

To conclude, current and/or foreseen experiments could measure an EDM for the neutron and/or electron and therefore provide evidence for CP violation and physics beyond the Standard Model. Such signals must be considered likely if supersymmetric unification is realized in nature. Furthermore, the combined effort of experiments on various systems can provide crucial information to distinguish the phisical source of the signal(s).

\section{References}

Altarev, I. S. et al., New measurement of the electric dipole moment of the neutron. Phys. Lett. , B276:242-246 (1992).

Barbieri, R., Ferrara, S., and Savoy, C. A., Gauge models with spontaneously broken local supersymmetry. Phys. Lett., 119B:343 (1982).

Barbieri, R. and Hall, L. J., Signals for supersymmetric unification. Phys. Lett. , B338:212-218 (1994).

Barbieri, R., Hall, L. J., and Strumia, A., Hadronic flavour and CP violating signals of superunification. Nucl. Phys., B449:437-461 (1995a).

Barbieri, R., Hall, L. J., and Strumia, A., Violations of lepton flavour and CP in supersymmetric unified theories. Nucl. Phys. , B445:219-251 (1995b).

Barbieri, R., Romanino, A., and Strumia, A., Electric dipole moments as signals of supersymmetric unification. Phys. Lett. , B369:283-288 (1996).

Barr, S. M., A review of CP violation in atoms. Int. J. Mod. Phys., A8:209-236 (1993).

Buchmuller, W. and Wyler, D., CP violation and R invariance in supersymmetric models of strong and electroweak interactions. Phys. Lett. , 121B:321 (1983).

Chamseddine, A. H., Arnowitt, R., and Nath, P., Locally supersymmetric grand unification. Phys. Rev. Lett. , 49:970 (1982).

Commins, E. D., Ross, S. B., DeMille, D., and Regan, B. C., Improved experimental limit on the electric dipole moment of the electron. Phys. Rev. , A50:2960 (1994).

\footnotetext{
${ }^{5}$ The errors come from the random sampling in the parameter space.
} 
del Aguila, F., Gavela, M. B., Grifols, J. A., and Mendez, A., Specifically supersymmetric contribution to electric dipole moments. Phys. Lett. , 126B:71 (1983).

DeMille, D., talk given at the Summer Institute on "Signals of unified theories", Gran Sasso (1995).

Dimopoulos, S. and Hall, L. J., Electric dipole moments as a test of supersymmetric unification. Phys. Lett. , B344:185-192 (1995).

Fischler, W., Paban, S., and Thomas, S., Bounds on microscopic physics from P and T violation in atoms and molecules. Phys. Lett. , B289:373-380 (1992).

Polkinski, J. and Wise, M. B., The electric dipole moment of the neutron in low-energy supergravity. Phys. Lett. , 125B:393 (1983).

Ramsey, N. F., In Proceedings of the Erice Summer School (1995).

Shabalin, E. P., The electric dipole moment of the neutron in a gauge theory. Sov. Phys. Usp. , 26:297 (1983). 